ISSN 1112-9867

Available online at

http://www.jfas.info

\title{
CONTRIBUTION OF ANTHROPIC ACTIVITIES TO THE SEAWATER POLLUTION AT THE PORT OF TENES
}

\author{
F. Guetarni ${ }^{1}$, M. Douani ${ }^{*}$, S. Kouadri Moustefai ${ }^{* 2}$ \\ ${ }^{1}$ Department of process engineering, Laboratory of Vegetal Chemistry, Water and Energy, \\ University Hassiba Benbouali, of Chlef,: Po. Box 151, Chlef, 02000, Algeria \\ ${ }^{2}$ Department of process engineering, Laboratory of Water and Environment, University \\ Hassiba Benbouali, of Chlef,: Po. Box 151, Chlef, 02000, Algeria
}

Received: 06 Mars 2017 / Accepted: 26 July 2017 / Published online: 01 September 2017

\begin{abstract}
The port of Ténès, located at West of Algiers, is a medium of intense anthropic activities. Its pollution was monitored using a sampling technique. The analysis of the parameters: $\mathrm{pH}$, temperature, electrical conductivity and dissolved oxygen (D.O) was carried out in situ. The concentration of heavy metals has been carried out by the atomic absorption while scanning the wavelengths varying from $217.0 \mathrm{~nm}$ at $228.8 \mathrm{~nm}$. The evolution of the nitrate and nitrite content was obtained by spectrophotometer. Their spatio-temporal profiles were obtained by the Geographic Information System (GIS) software. The high toxicity of seawater is due to the presence of lead and cadmium with concentrations exceeding 60 times the normal value $(0.015 \mathrm{mg} / \mathrm{l})$. Moreover, the deficit in D.O announces strong biological activities. This wild aggression is related to the industrial discharges in the port area.
\end{abstract}

Keywords:marine pollution; heavy metals;sampling; physicochemical analysis; toxicity.

AuthorCorrespondence, e-mail:douani_mustapha@yahoo.com

doi: http://dx.doi.org/10.4314/jfas.v9i3.11 


\section{INTRODUCTION}

A l'instar des pays africains touchés par le stress hydrique, l'Algérie se situe dans la catégorie des pays les plus pauvres en matière de potentialités hydriques, soit en dessous du seuil théorique de rareté, fixé par la Banque Mondiale, à $1000 \mathrm{~m}^{3}$ par habitant et par an. L’Organisation Mondiale de la Santé (O.M.S) prévoit une régression de la disponibilité en eau potable en Algérie pour avoisiner légèrement le seuil des $400 \mathrm{~m}^{3} /$ habitant/an. Le tableau I est le récapitulatif de l'évolution des disponibilités en eau durant les quatre dernières décennies.

Tableau 1. Evolution de la disponibilité en eau théorique par habitant en Algérie [1]

\begin{tabular}{lccccc}
\hline Année de référence & 1962 & 1990 & 1995 & 1998 & 2020 \\
\hline $\begin{array}{l}\text { Disponibilité en eau théorique } \\
\text { (m³/hab/an.) }\end{array}$ & 1500 & 720 & 680 & 630 & 430 \\
\hline
\end{tabular}

Pour combler ce déficit, il y a lieu de mobiliser toutes les ressources d'eau localement disponibles pour sécuriser l'alimentation en eau potable et de répondre aux besoins croissantsde l'agriculture. De ce fait, le milieu marin représente un enjeu considérable en termes de développements socioéconomiques surtout que le littoral algérien s'étale sur une longueur de $1200 \mathrm{~km}$.

Le dessalement de l'eau de mer qui reste une perspective pour répondre aux problèmes d'approvisionnement des populations en eau potable, nécessite à la fois le choix d'un procédé adéquat de dessalement et d'un site le moins pollué. Cette dernière exigence passe impérativement par des analyses physiques, chimiques, et bactériologiques de l'eau des sites potentiels de façon quasi périodique. La dégradation de la qualité d'eau de mer est intimement liée aux rejets urbains et industriels vers la mer. Cette contamination d'eau de mer est la principale cause de la détérioration de l'écosystème côtier en Algérie (Laib \& Leghouchi 2012; Belabed et al. 2013; Tayeb et al. 2015; Kouidri et al. 2016; Benmecheta \& Belkhir 2016)[2-6]. Dans cette perspective et compte tenu de l'activité humaine et industrielle du port de Ténès, l'étude de l'évolution de la contamination du milieu marin par des substances toxiques, nécessite la mise en place d'une politique de contrôles périodiques par des analyses des eaux marines en vue d'éviter les retombées liées à la présence d'agents polluants, avec des 
concentrations qui dépasseraient le seuil de toxicité.

\subsection{Zone d'étude (port de Ténès)}

Le port de Ténès, ancré dans la petite baie de Ténès, est situé à $240 \mathrm{~km}$ à l'Ouest d'Alger, a comme coordonnées $36^{\circ} 32^{\prime}$ de latitude Nord et $01^{\circ} 32^{\prime}$ de longitude Est. Le port est formé par deux jetées (Est de 400 m.l (mètre linéaire) et Ouest de $700 \mathrm{~m} .1$ ) séparées par une ouverture de $160 \mathrm{~m}$, et protégé par un brise-lame de $450 \mathrm{~m}$. La surface du plan d'eau est de 20 ha avec des profondeurs de $12 \mathrm{~m}$ à la passe Est et $14 \mathrm{~m}$ à la passe Ouest.

Bien que le Port de Ténès soit construit entre 1868 - 1907, il est considéré comme un site pour les activités commerciales, de pêche et de plaisance. L'oued Alala, qui débouche approximativement prés du port de Ténès, véhicule d'intenses rejets industriels et urbains. La position géographique du port, cible de notre analyse, est présentée à travers la figure 1.

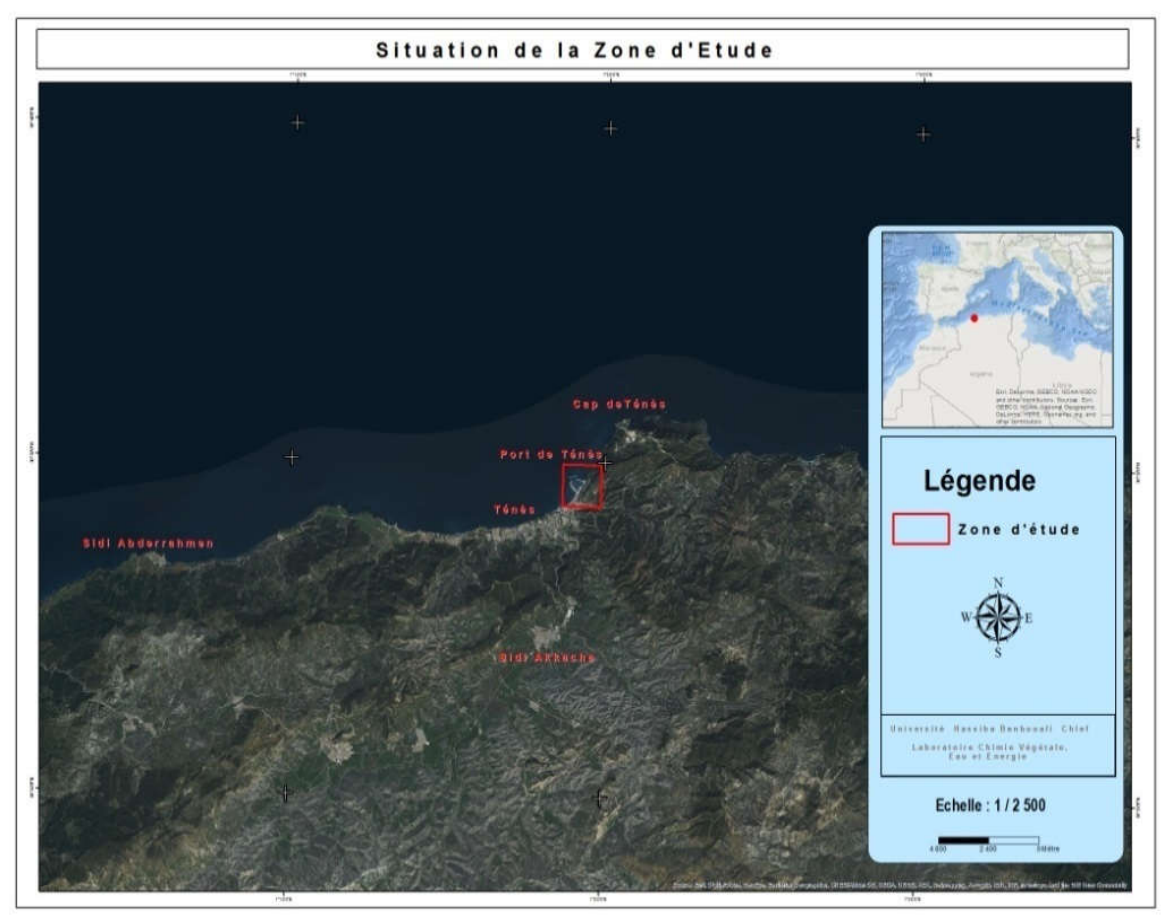

Fig.1. Position géographique du site ciblé. (Port de Ténès). 


\subsection{Localisation des points de prélèvement:}

Pour procéder à une analyse exhaustive permettant la détermination du degré de pollution du site, nous avons opté pour une stratégie de balayage en fixant les points de prélèvement conformément au schéma et aux figures 2 et 3 , présentés ci-après:

\section{La mer}

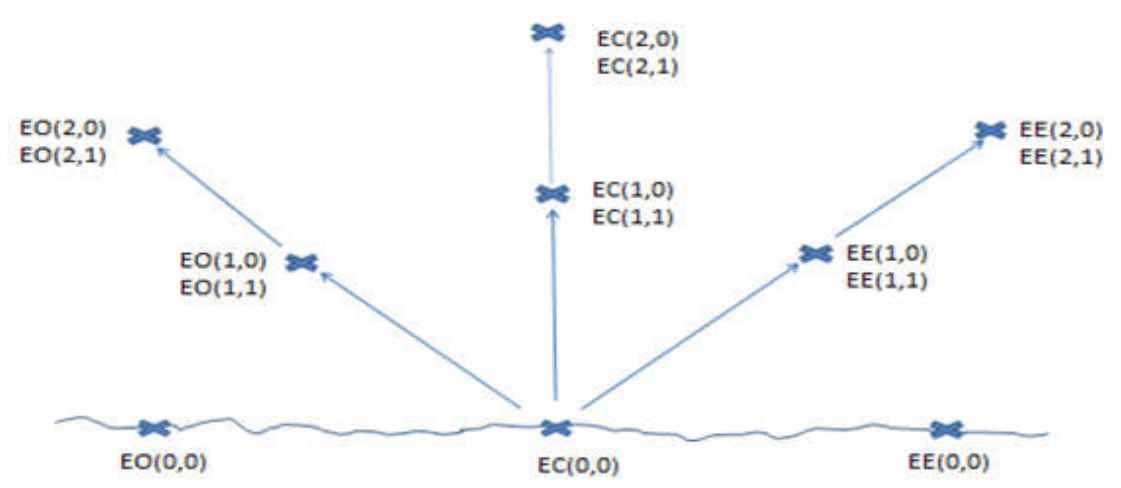

Fig.2. Schéma de répartition des points d'échantillonnage.
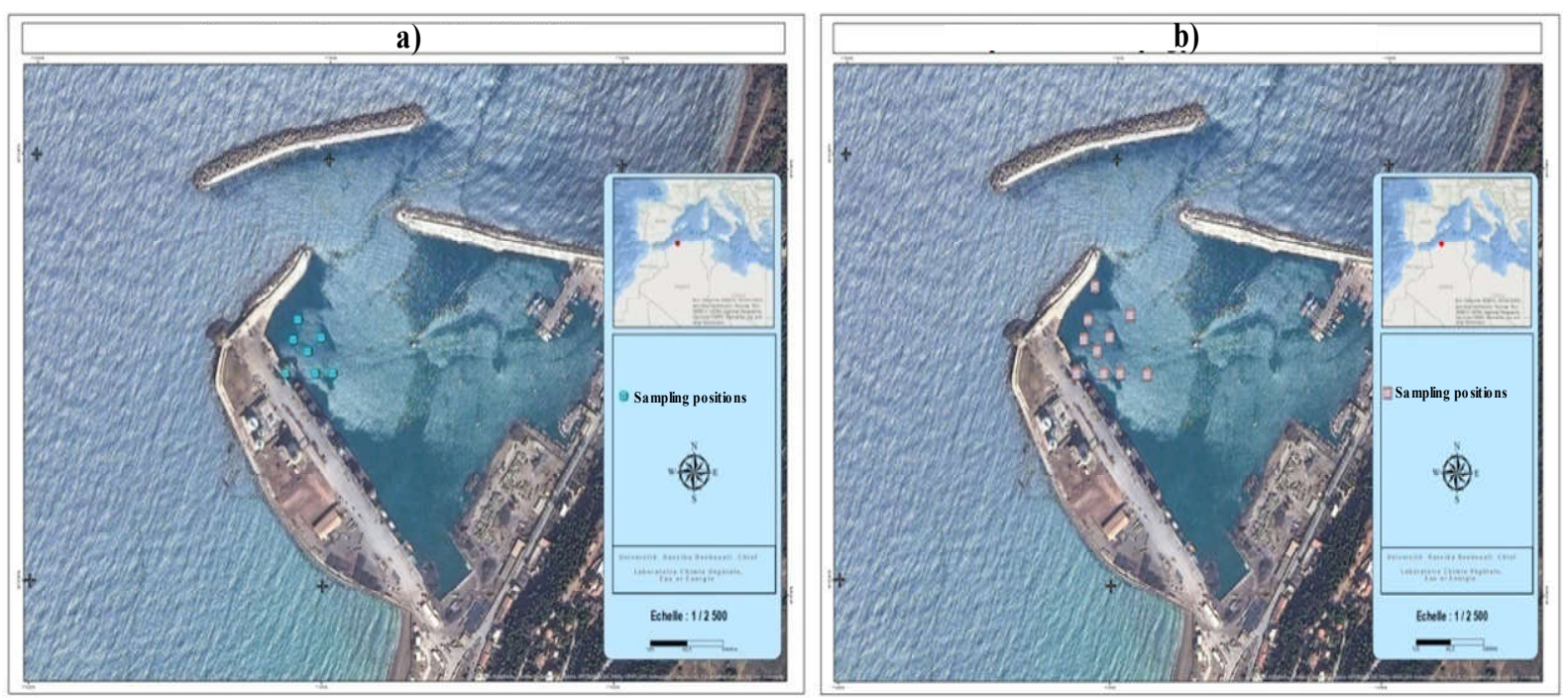

Fig.3. Répartition des points et périodes de prélèvements.
a)- Mai 2014
b)- Decembre 2014

La disposition de ces points est en rapport avec la circulation des courants marins dans le site d'une part et du point de rejets des effluents industriels et ménagers des agglomérations 
locales d'autre part. Ils sont espacés suivant un rapport égal au nombre d'or tels que présentés sur la figure 2. Ils sont identifiés comme suit :

- $\quad 0$ : Le point à la surface libre du point de prélèvement $(0 \mathrm{~m})$.

- $\quad 1$ : Le point le plus profond du point de prélèvement $(7 \mathrm{~m})$.

- $\quad$ EC : Le point central.

- $\quad$ EE : Le point Est par rapport au point de référence.

- $\quad$ EO : Le point Ouest par rapport au point de référence.

On note que la largeur des intervalles suivants :

$[\mathrm{EC}(0,0), \mathrm{EE}(0,0)],[\mathrm{EC}(0,0), \mathrm{EO}(0,0)],[\mathrm{EC}(0,0), \mathrm{EE}(1,0)],[\mathrm{EC}(0,0), \mathrm{EC}(1,0)],[\mathrm{EC}(0,0)$, $\mathrm{EO}(1,0)]$ est de 50m, alors pour celle des intervalles [EC(0,0), $\operatorname{EE}(2,0)],[\operatorname{EC}(0,0), \operatorname{EE}(2,0)]$, $[\mathrm{EC}(0,0), \mathrm{EC}(2,0)],[\mathrm{EC}(0,0), \mathrm{EO}(2,0)]$ la distance est de $80 \mathrm{~m}$.

Afin d'accomplir des analyses durant le mois décembre 2014, d'autres points comme indiqué dans les positions suivantes : [EC(0,0), EE (3.0)], [EC (0,0), EC (3.0)], [EC (0.0) EO (3.0)] ont été ajoutés avec une largeur d'intervalles égale à $130 \mathrm{~m}$.

Les points de prélèvement se situent dans un domaine où l'activité portuaire de navires de marchandises est très importante vu l'importante contribution de ces derniers à la pollution de l'écosystème.

\section{TECHNIQUES EXPÉRIMENTALES}

\subsection{Appareillage d'analyse des échantillons}

Le prélèvement des échantillons de l'eau de mer s'est effectué aux différents endroits (en surface et en profondeur) à l'aide des flacons en verre, de capacité $200 \mathrm{ml}$, fermés avec des bouchons en liège. Ces échantillons sont destinés à l'analyse des paramètres physico-chimiques, paramètres indicateurs de la pollution. Les paramètres physiques (température, $\mathrm{pH}$, salinité, conductivité et oxygène dissous (O.D)) sont mesurés in-situ à l'aide d'une valise multiparamètres de type (SX 736). Les paramètres chimiques, tels que les concentrations en métaux lourds, sont mesurés au laboratoire, par l'application de la technique de l'absorption atomique sur un appareil du type (SAA Varion X220) et pour longueurs d'ondes appropriées, $217,0 \mathrm{~nm}$ et $228,8 \mathrm{~nm}$ pour le plomb et cadmium 
respectivement. Les nitrites et les nitrates sont analysés par un photomètre du type (Loviband model).

\section{MESURE DES PARAMETRES PHYSICO-CHIMIQUES}

\subsection{L'oxygène dissous (O.D).}

L'oxygène moléculaire dissous est une caractéristique importante du milieu qui gouverne la majorité des phénomènes chimiques et biologiques de l'écosystème marin [7]. Sa concentration est la résultante de l'interaction de facteurs physiques (température, échange air-mer et la salinité), chimiques (oxydation chimique) et biologiques (photosynthèse, respiration et régénération) [8]. Elle peut subir des fluctuations qui dépendent de la température, de la salinité de l'eau, de la pression atmosphérique et de la température de l'air ambiant. Si l'oxygène dissous semble peu influer sur la concentration en polluants, une teneur élevée conduit au développement biologique des espèces marines qui s’y trouvent exposées via leurs appareils respiratoires, à un transfert actif de polluants dans la chaine alimentaire [9] Pour les périodes de mois de mai et de décembre, les résultats relevés au niveau des différents points ciblés sont présentés après traitement par le logiciel (S.I.G), sous forme d'iso-concentrations en oxygène dissous, donnés par les figures 4 et 5 .

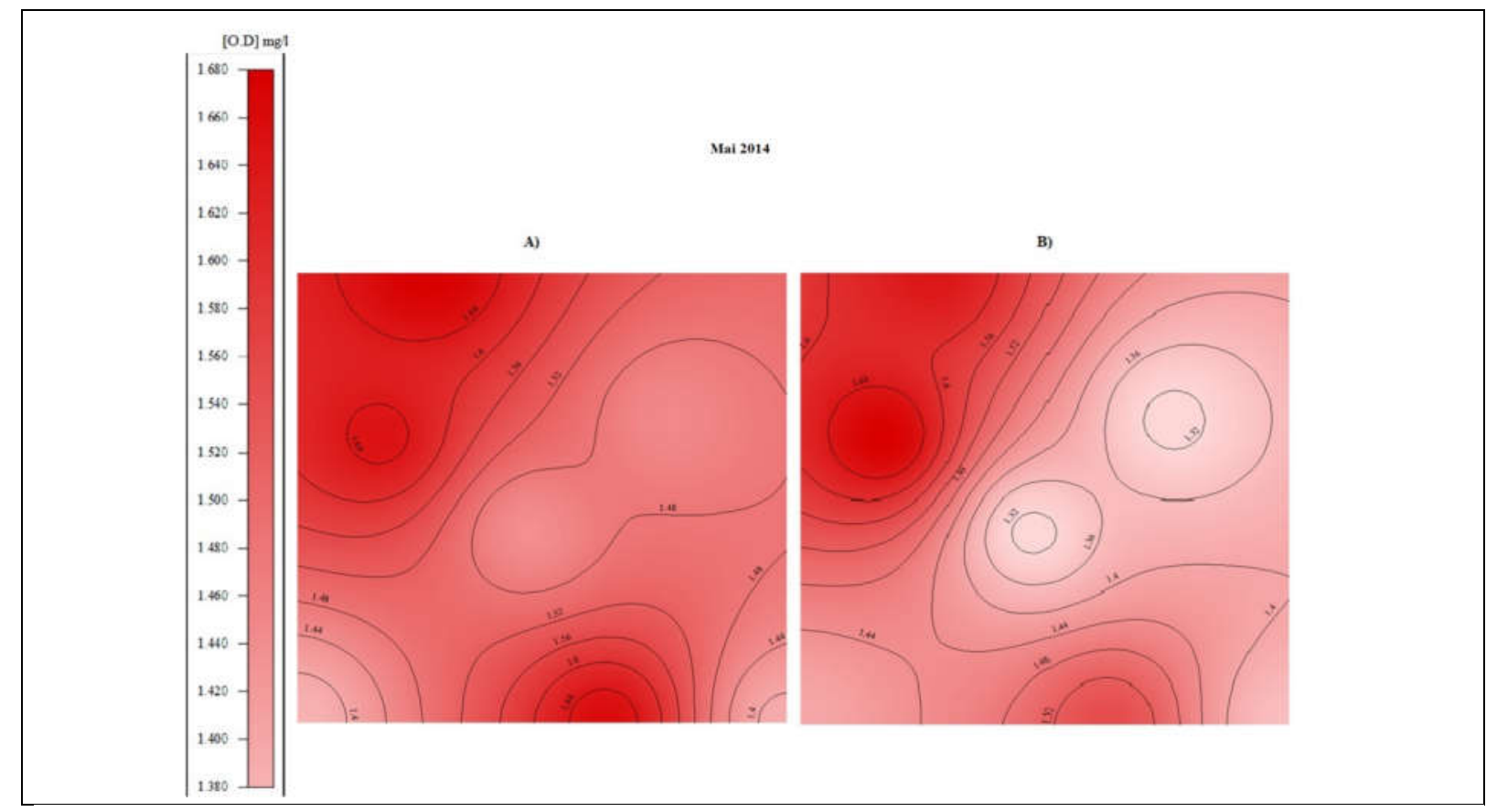

Fig.4. Distribution spatiale de l'oxygène dissous dans le port de Ténès, Mai 2014 :

A) Surface libre ; B) A $7 \mathrm{~m}$ de profondeur 


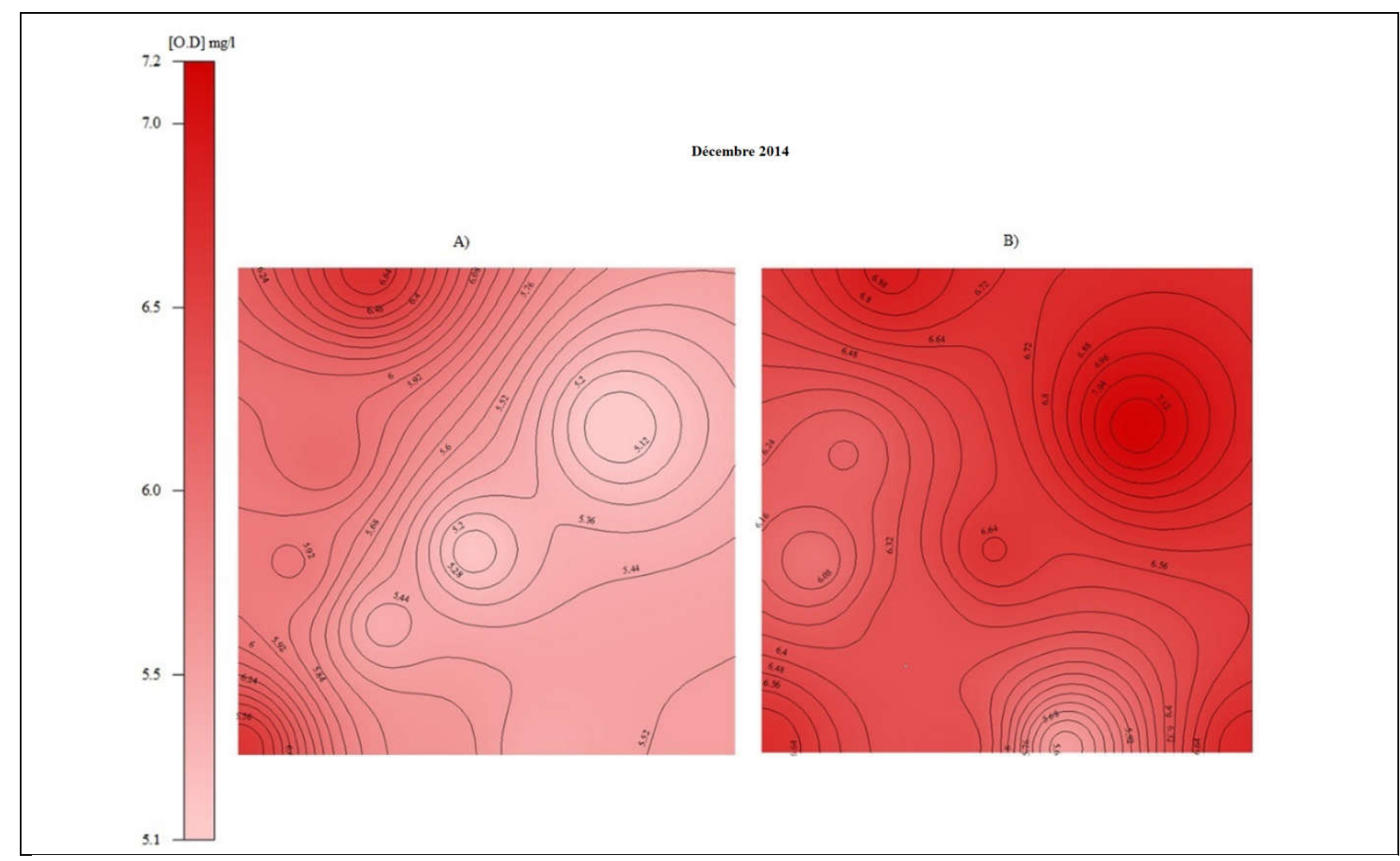

Fig.5. Distribution spatiale de l'oxygène dissous dans le port de Ténès, Décembre 2014 :

A) Surface libre ; B) A $7 \mathrm{~m}$ de profondeur.

Durant la période du mois de mai, on note que la teneur en oxygène dissous, à la surface libre, est relativement plus importante que pour les différentes profondeurs. Cette distribution spatiale est liée à la solubilité de l'oxygène dans l'eau. En effet, la couche superficielle de l'eau constitue, hypothétiquement, un film très riche en oxygène qui diminue avec la profondeur dont le gradient transversal $\left(\frac{d\left[\mathrm{O}_{2}\right]}{d z}\right)$ est fonction des paramètres liés principalement à l'activité biologique et à certains processus chimiques (oxydation) [10]. Des études antérieures ont corrélé la solubilité de l'oxygène à la température et à la salinité via l'équation de Benson et Krause (1984)[11].

$$
C^{\circ}=\exp (A(T)) * \exp (-B(T) * S)
$$

où $\mathrm{A}(\mathrm{T})$ et $\mathrm{B}(\mathrm{T})$ sont des fonctions de la température dont les expressions sont données par l'équation de Benson et Krause (1984): 


$$
\begin{gathered}
A(T)=-135.29996+\frac{1.572288 * 10^{5}}{T}-\frac{6.637149 * 10^{7}}{T^{2}}+\frac{1.243678 * 10^{10}}{T^{3}} \\
-\frac{8.621061 * 10^{11}}{T^{4}} \\
B(T)=-0.020579-\frac{12.142}{T}+\frac{2.3631 * 10^{3}}{T^{2}}
\end{gathered}
$$

On peut déceler les effets conjugués et opposés de la température et de la salinité $\mathrm{S}$, sur la solubilité de l'oxygène. A salinité constante, on peut noter que la concentration en oxygène dissous est une fonction décroissante de la température [12]. Sur la plage de températures relevées, les concentrations en O.D sont données par le tableau 2.

Tableau 2: Solubilité de l'oxygène en fonction de la température et de la salinité.

\begin{tabular}{lllllll} 
& \multicolumn{7}{c}{$\mathbf{S = 3 5 \%}$} \\
\hline $\mathrm{T}\left({ }^{\circ} \mathrm{C}\right)$ & 15 & 18 & 21 & 24 & 27 & 30 \\
\hline O.D $(\mathrm{mg} / \mathrm{l})$ & 7,927 & 7,484 & 7,087 & 6,728 & 6,401 & 6,102 \\
\hline
\end{tabular}

Pour l'eau de mer de la baie de Ténès et lors du mois de décembre, les conditions de température était de $17^{\circ} \mathrm{C}$ avec une salinité de $35 \%$, alors la valeur de la solubilité relevée est en parfait accord avec celle prédite par l'équation de Benson et Krause (1984), et qui est de 238,33 $\mu \mathrm{molO}_{2} / \mathrm{Kg}(7,63 \mathrm{ppm})$. Or, l'eau de mer peut être sursaturée ou sous-saturée en oxygène, selon les processus physiques, chimiques et biologiques. On définit le taux de saturation par:

$$
\% S A T=\frac{C_{\max }-C_{\min }}{C_{\max }} * 100
$$

Une analyse de données relative au profil de la concentration en oxygène dissous en fonction de la profondeur a établi qu'à une profondeur $\mathrm{h}=7 \mathrm{~m}$, la concentration en oxygène dissous est de $6,47 \mathrm{mg} / 1$ lorsque $\mathrm{T}=17^{\circ} \mathrm{C}$, alors qu'elle est de $1,46 \mathrm{mg} / 1$ à $\mathrm{T}=22^{\circ} \mathrm{C}$ soit un taux de saturation de $83,49 \%$ et $20,66 \%$ respectivement.

De tels résultats laissent augurer la présence d'une forte activité biologique dans les strates les plus illuminées avec d'éventuelles réactions chimiques dégageant de l'oxygène (photosynthèse) avec la présence de Matière En Suspension (M.E.S). Si durant la période de 
décembre $\left(\mathrm{T}=17^{\circ} \mathrm{C}\right)$, la teneur en O.D répond parfaitement aux normes de qualité d'eau, fixées à un taux de saturation de l'ordre de 80-120\%, un déficit flagrant en O.D est à mentionner pour la période du mois de mai. De tels cas laissent entrevoir la nécessité impétueuse de l'intervention de l'homme pour limiter le taux de pollution de milieu marin [13].

Par ailleurs, la profondeur de pénétration du rayon lumineux est en accord parfait avec les concentrations en $\mathrm{O}_{2}$ et en $\mathrm{CO}_{2}$ dans le fond marin liée au phytoplancton. Du point de vue transfert de molécules d'oxygène, on note qu'un renouvellement intense par ventilation favorise le coefficient de transfert de l'oxygène vers les couches stratifiées du lit liquide via l'équation(Chen et al. 1976) [14].

$$
K_{L}=\frac{86400 * \mathrm{D}}{(200-60 \sqrt{V}) 10^{-6}}
$$

Avec $\mathrm{D}\left(\mathrm{m}^{2} / \mathrm{sec}\right)$ et $\mathrm{V}(\mathrm{m} / \mathrm{sec})$, coefficient de diffusion de l'oxygène et vitesse du vent respectivement. Les études de phénomènes de transfert établissent clairement l'effet positif de la température sur le coefficient de diffusion $\mathrm{D}$ et la viscosité de l'eau $\mu$.

\subsection{La température (T).}

C'est une caractéristique physique importante qui, en plus de son impact négatif sur la teneur en oxygène dissous, affecte positivement la solubilité des sels via l'équation $[15,16]$.

$$
S=(0.72 * \sigma-3.06) *(1+0.02 *(T-25))
$$

Cette grandeur est liée à la présence d'espèces ioniques dissoutes dans le milieu marin (bromures, iodures, chlorures, matière organique oxydée).

En outre, le réchauffement de l'eau est responsable de l'accélération des processus métaboliques conditionnés par un accroissement de l'absorption des métaux lourds. Toutefois, une augmentation de la température en surface se répercute par les courants convectifs de masse d'eau entre les différentes strates de l'eau de mer. Il en découle que la détermination du champ de température est nécessaire pour étudier les transferts de matière entre les différentes strates de l'eau de mer et le fond marin. Dans ce cadre, les mesures relatives aux mois de mai et de décembre sont présentées sous forme d'iso thermes sur les figures 6 et 7 . 


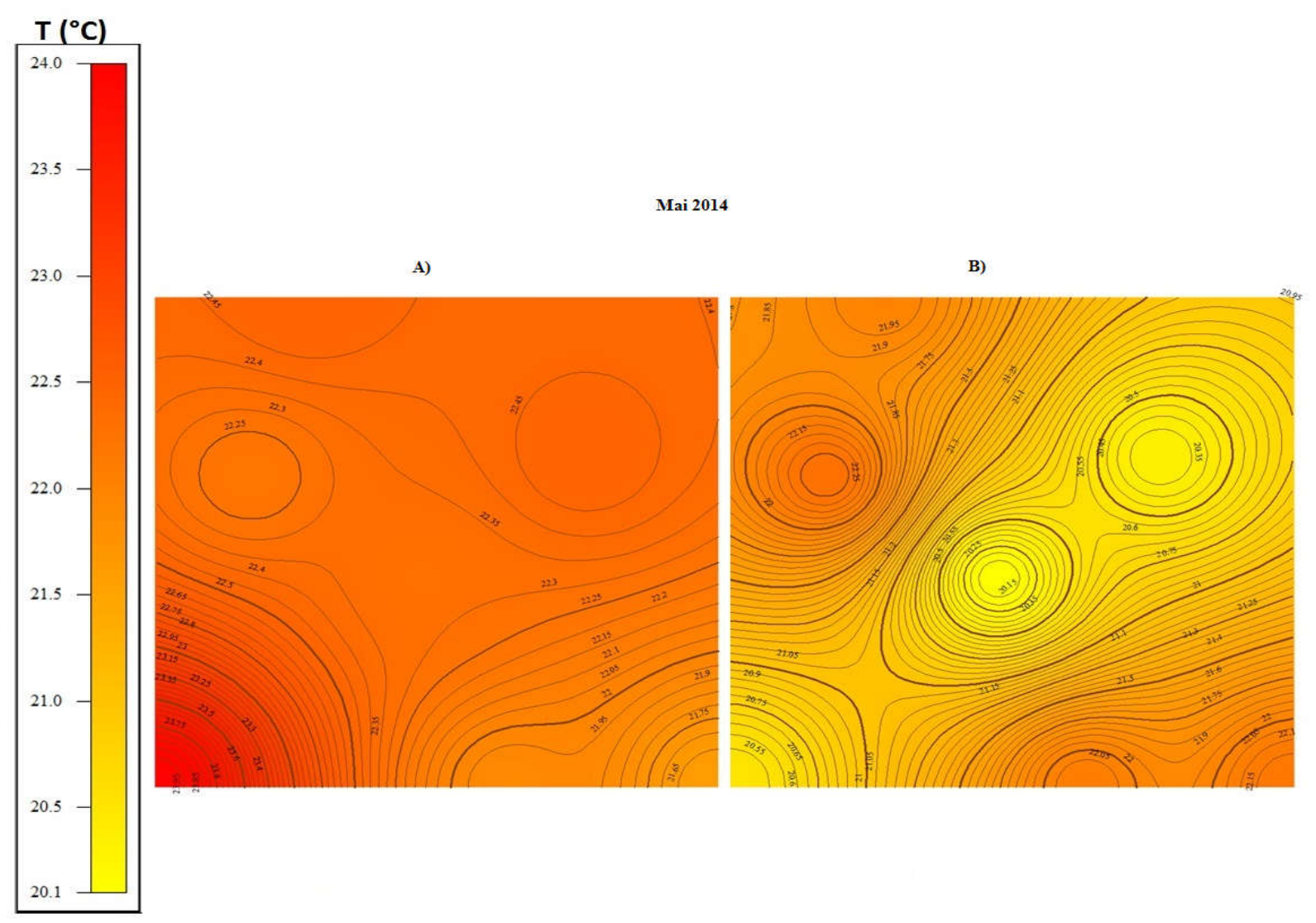

Fig.6. Distribution spatiale de la température dans le port de Ténès, Mai 2014 :

A) Surface libre ; B) A $7 \mathrm{~m}$ de profondeur. 


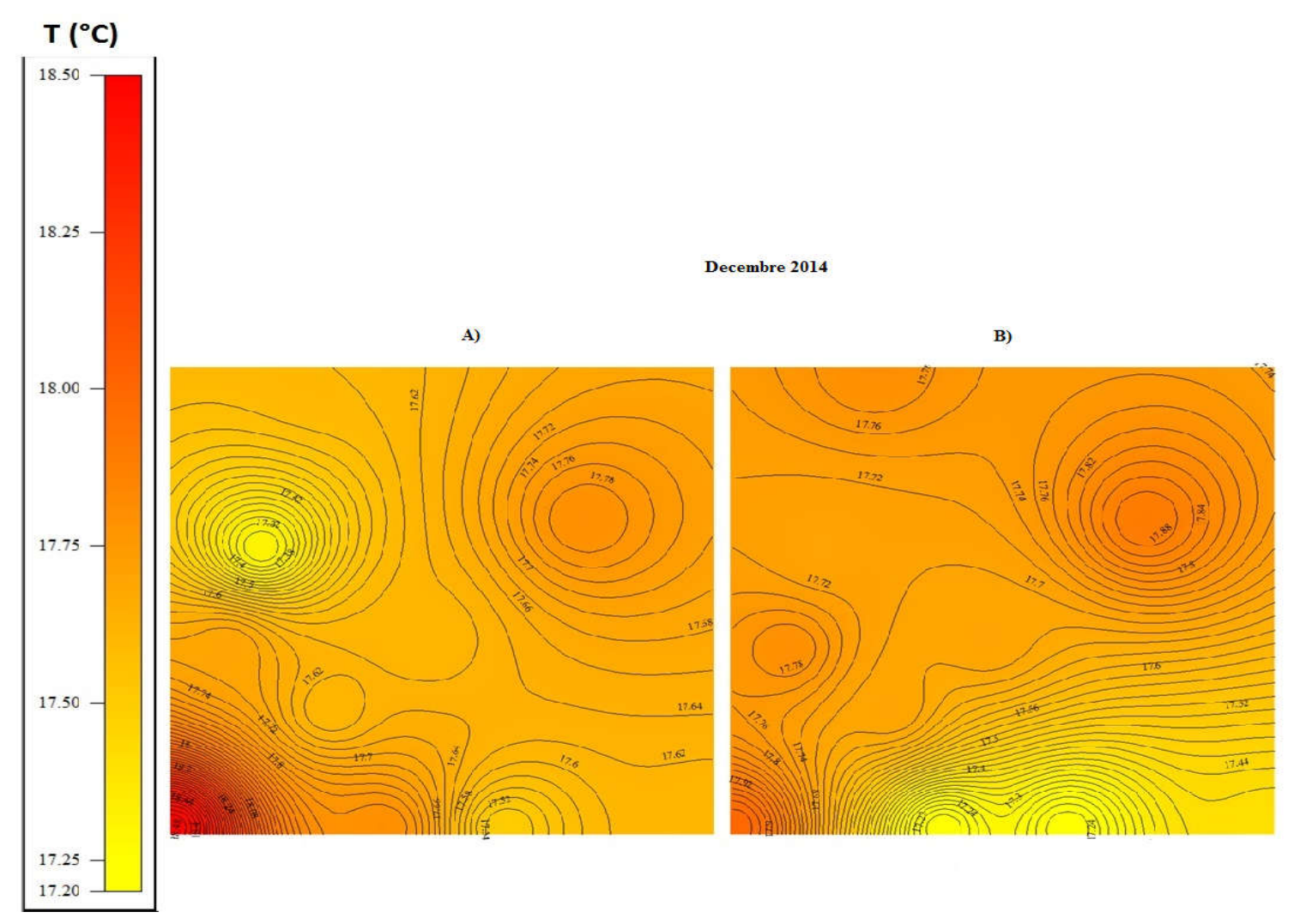

Fig.7. Distribution spatiale de la température dans le port de Ténès, Décembre 2014 :

A) Surface libre ; B) A $7 \mathrm{~m}$ de profondeur.

L'étude cartographique par logiciel SIG (version 10.3), nous a permis de tracer des isothermes aussi bien en surface qu'à tout niveau de la strate dans le milieu marin en vue de situer la direction des déplacements locaux des flux marins dont les conséquences sont la dispersion des espèces chimiques.

Les résultats relevés durant le mois de mai (2014) montrent qu'il y a une légère fluctuation de la température entre les différents points de la zone d'étude. Cette tendance vers l'uniformisation de la distribution de température est due au brassage de courants marins entre les différentes strates de l'eau. Ce mouvement d'advection (diffusion et convection) demeure dépendant d'un gradient de masse volumique lequel est intimement lié au profil de température à travers une section longitudinale.

Si les relevés météorologiques ont noté que la température de l'air ambiant est de $18^{\circ} \mathrm{C}$ avec une vitesse de vent de $(8,33 \mathrm{~m} / \mathrm{sec})$, on peut avancer qu'un phénomène d'évaporation de l'eau prend naissance en surface pour tendre à un équilibre thermique entre l'air ambiant et la 
surface libre de l'eau soit un écart moyen de $\frac{\Delta T}{\Delta x}=-\frac{1,83^{\circ} \mathrm{C}}{6 \mathrm{5n}}$, indépendamment du point de prélèvement.

Compte tenu de la faible agitation du milieu marin, on note que les couches profondes préservent leur niveau de température autour de $22^{\circ} \mathrm{C}$. En outre, les températures relevées en mois de décembre (2014) sont pratiquement constantes $\left(17,6^{\circ} \mathrm{C}\right)$ aussi bien en surface qu'à différentes profondeurs de la zone d'étude.

\subsection{Les nitrites et les nitrates}

L'azote Kjeldhal est une source potentielle de production de nitrates dans l'eau. Les normes OMS fixent à $50 \mathrm{mg} / \mathrm{l}$ la concentration de nitrates dans les eaux de consommation [17].

En général, la variation de la concentration en azote est difficile à délimiter compte tenu de ses formes organiques et minérales. Il est souvent réduit par les algues.

$$
\mathrm{NO}_{3}^{-} \rightarrow \mathrm{NO}_{2}^{-} \rightarrow \mathrm{NH}_{4}^{+} \rightarrow \mathrm{N}_{2} \uparrow
$$

Ayant rapport aux activités agricoles (engrais), la concentration en nitrates $\left(\mathrm{NO}_{3}{ }^{-}\right)$a tendance à augmenter. La fixation de l'azote gazeux dans l'eau est réalisée par les différents organismes phytosynthétiques. Toutefois, en condition anaérobie, la réduction bactérienne contribue à la formation des oxydes d'azotes $\left(\mathrm{NO}, \mathrm{N}_{2} \mathrm{O}\right)$. En toute rigueur, l'azote est un facteur limitant de la production phytoplanctonique.

Les résultats d'analyses des échantillons prélevés ont montré que la concentration en nitrites aussi bien en surface qu'en profondeur reste très faible $(\approx 0,02 \mathrm{mg} / \mathrm{l})$. Toutefois, une légère augmentation en nitrites en profondeur est attribuée à l'activité microbienne qui se trouve corrélée à une augmentation de la température durant le mois de mai.

Les résultats sont présentés dans les tableaux 3 et 4 où on note une distribution quasi uniforme de la concentration des nitrites.

Tableau 3. Distribution de nitrites et nitrates en Mai 2014

\begin{tabular}{ccc}
\hline Points de prélèvements & Nitrites $(\mathrm{mg} / \mathrm{l})$ & Nitrates $(\mathrm{mg} / \mathrm{l})$ \\
\hline $\mathrm{EC}(0,0)$ & 0,080 & 0,269 \\
$\mathrm{EC}(0,1)$ & $<0,01$ & $<0,01$ \\
$\mathrm{EC}(1,0)$ & 0,060 & 0,197 \\
$\mathrm{EC}(1,1)$ & 0,020 & 0,065
\end{tabular}




\begin{tabular}{lcc}
$\mathrm{EC}(2,0)$ & $<0,01$ & $<0,01$ \\
$\mathrm{EC}(2,1)$ & $<0,01$ & $<0,01$ \\
$\mathrm{EE}(1,0)$ & $<0,01$ & $<0,01$ \\
$\mathrm{EE}(1,1)$ & 0,020 & 0,066 \\
$\mathrm{EE}(2,0)$ & 0,050 & 0,164 \\
$\mathrm{EE}(2,1)$ & $<0,01$ & $<0,01$ \\
$\operatorname{EO}(1,0)$ & 0,020 & 0,065 \\
$\operatorname{EO}(1,1)$ & $<0,01$ & $<0,01$ \\
$\operatorname{EO}(2,0)$ & 0,030 & 0,098 \\
$\operatorname{EO}(2,1)$ & $<0,01$ & $<0,01$ \\
\hline
\end{tabular}

Tableau IV. Distribution de nitrites et nitrates en Décembre 2014.

\begin{tabular}{|c|c|c|}
\hline Points de prélèvements & Nitrites (mg/l) & Nitrates $(\mathrm{mg} / \mathrm{l})$ \\
\hline $\mathrm{EC}(0,0)$ & 0,02 & 0,065 \\
\hline $\mathrm{EC}(0,1)$ & 0,02 & 0,065 \\
\hline $\mathrm{EC}(1,0)$ & 0,02 & 0,065 \\
\hline $\mathrm{EC}(1,1)$ & $<0,01$ & $<0,01$ \\
\hline $\mathrm{EC}(2,0)$ & $<0,01$ & $<0,01$ \\
\hline $\mathrm{EC}(2,1)$ & 0,02 & 0,065 \\
\hline $\mathrm{EC}(3,0)$ & 0,02 & 0,065 \\
\hline $\mathrm{EC}(3,1)$ & 0,02 & 0,065 \\
\hline $\mathrm{EE}(1,0)$ & 0,02 & 0,065 \\
\hline $\mathrm{EE}(1,1)$ & 0,01 & 0,033 \\
\hline $\mathrm{EE}(2,0)$ & 0,02 & 0,065 \\
\hline $\mathrm{EE}(2,1)$ & $<0.01$ & $<0.01$ \\
\hline $\mathrm{EE}(3,0)$ & 0,02 & 0,065 \\
\hline $\mathrm{EE}(3,1)$ & $<0.01$ & $<0.01$ \\
\hline $\mathrm{EO}(1,0)$ & 0,02 & 0,065 \\
\hline $\mathrm{EO}(1,1)$ & $<0.01$ & $<0.01$ \\
\hline
\end{tabular}




$\begin{array}{lcc}\mathrm{EO}(2,0) & 0,01 & 0,033 \\ \mathrm{EO}(2,1) & <0.01 & <0.01 \\ \mathrm{EO}(3,0) & 0,01 & 0,033 \\ \mathrm{EO}(3,1) & <0,01 & <0,01\end{array}$

Les autres formes de l'azote et particulièrement les nitrates ont exhibé des concentrations moyennes de l'ordre de $(\approx 0,065 \mathrm{mg} / \mathrm{l})$. Un tel résultat confirme la corrélation développée dans les travaux de Robertson et al. [18].

\subsection{Les métaux lourds}

Les métaux lourds sont des polluants sérieux de l'environnement aquatique vu leur toxicité, leur persistance et leur lente biodégradabilité ainsi que leur tendance à se concentrer dans les organismes aquatiques

En vue de contribuer à l'établissement de profils spatio-temporels de concentration de métaux lourds $(\mathrm{Pb}, \mathrm{Cd}$, etc..) sur les côtes algériennes, nous avons analysé par absorption atomique SAA- Varion X220 les échantillons prélevés aux différents points prévus dans le cadre de cette étude

\subsubsection{Le plomb $(\mathrm{Pb})$}

Au-dessus d'un seuil de $100 \mu \mathrm{g}$ par litre de sang, le plomb est responsable du saturnisme [19].Sachant qu'il n'est pas totalement soluble dans l'eau de mer et compte tenu de sa masse volumique importante, il s'accumule en sédiments. Cependant, il y a lieu de mentionner que la turbulence des courants marins conduit probablement à la dispersion $\mathrm{du} \mathrm{Pb}$ dans les sédiments [20]. Les résultats des analyses des échantillons prélevés sont consignés dans les figures 8 et 9 . 


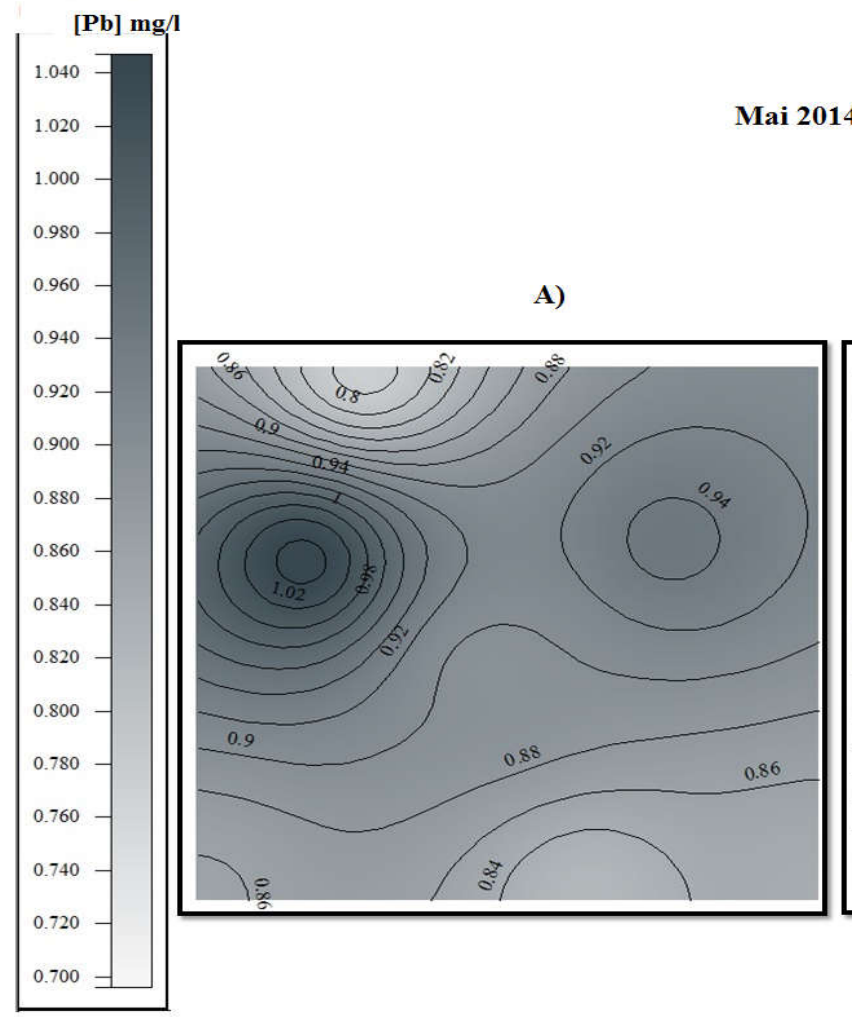

Fig.8. Distribution spatiale de la concentration du plomb dans le port de Ténès, Mai 2014 :

A) Surface libre ; B) A $7 \mathrm{~m}$ de profondeur

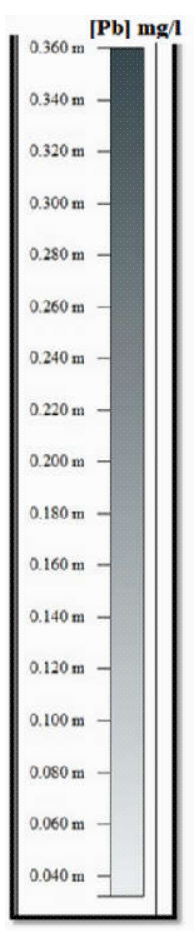

Décembre 2014

A)

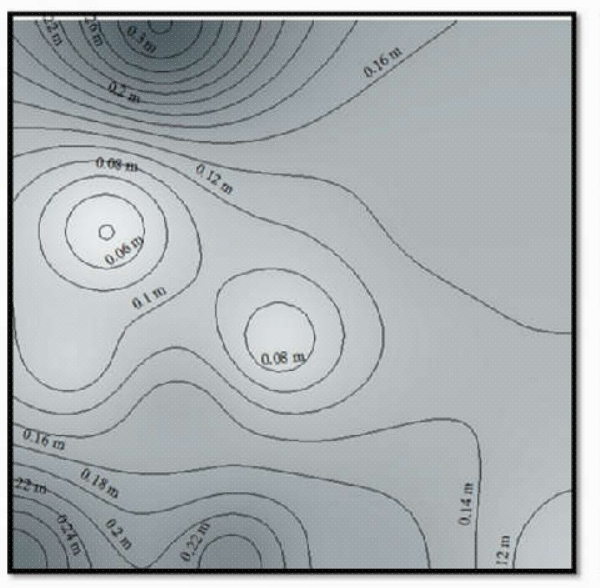

B)

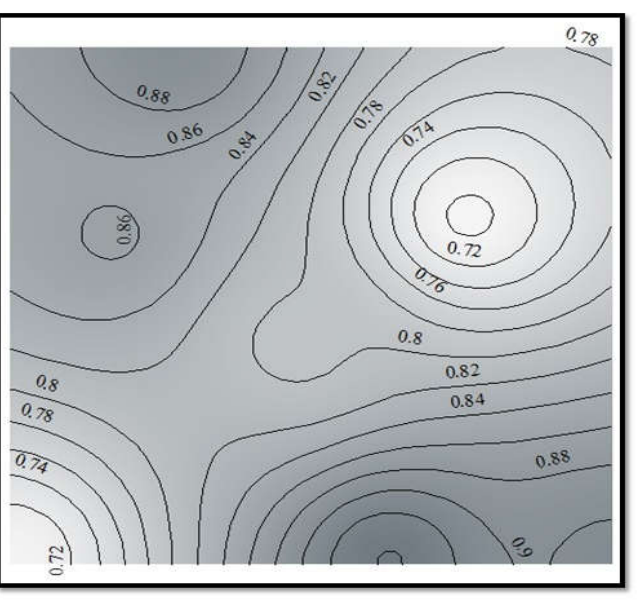

Fig.9. Distribution spatiale de la concentration du plomb dans le port de Ténès, Décembre 2014 :

A) Surface libre ; B) A $7 \mathrm{~m}$ de profondeur 
Il y a lieu de mentionner que dans les conditions naturelles, la concentration critique est de l'ordre $0,015 \mathrm{mg} / 1[21]$.

Les fortes concentrations révélées sont corrélées à :

- Rejets de l'industrie de CERAMIT qui sont composés de produits de l'émaillage de pièces sanitaires.

- Emissions dans l'air des produits de la combustion dans les moteurs à explosion

- Les rejets ménagers issus de la dégradation des vieilles conduites de plomb.

Pour dégager une idée très claire, on a présenté sur les courbes iso-concentration en plomb à différents niveaux sur les figures 8 et 9 En effet, on note une forte pollution en matière de plomb laquelle est de 60 fois plus grande de celle la concentration ordinaire.

Cette constatation est en parfaite adéquation avec la contribution de rejets susmentionnés. Relativement aux normes algériennes $(0,50 \mathrm{mg} / \mathrm{l})$, on note que la concentration en $\mathrm{Pb}$ est très élevée. Outre cet aspect, la concentration en $\mathrm{Pb}$ dans les sédiments explique en partie la forte quantité de plomb dispersée dans le milieu marin [22]. Ces niveaux élevés en concentrations seraient des risques de santé publique s'il est introduit dans la chaine alimentaire

\subsubsection{Le cadmium (Cd)}

C'est un métal de masse volumique égale à $\rho=8690 \mathrm{~kg} / \mathrm{m}^{3}$. Il a tendance à s'accumuler au niveau de la vase (sédiment). Certains cas d'intoxication par le cadmium ont été signalés JOCE 2004 (Official Journal of the EuropeanCommunities).

Pour les normes de l'OMS, la dose hebdomadaire acceptable est $7 \mu \mathrm{g} / \mathrm{kg} / \mathrm{semaine}$. Sa concentration en milieu aqueux est corrélée à la salinité ainsi qu'à la température [23]. 


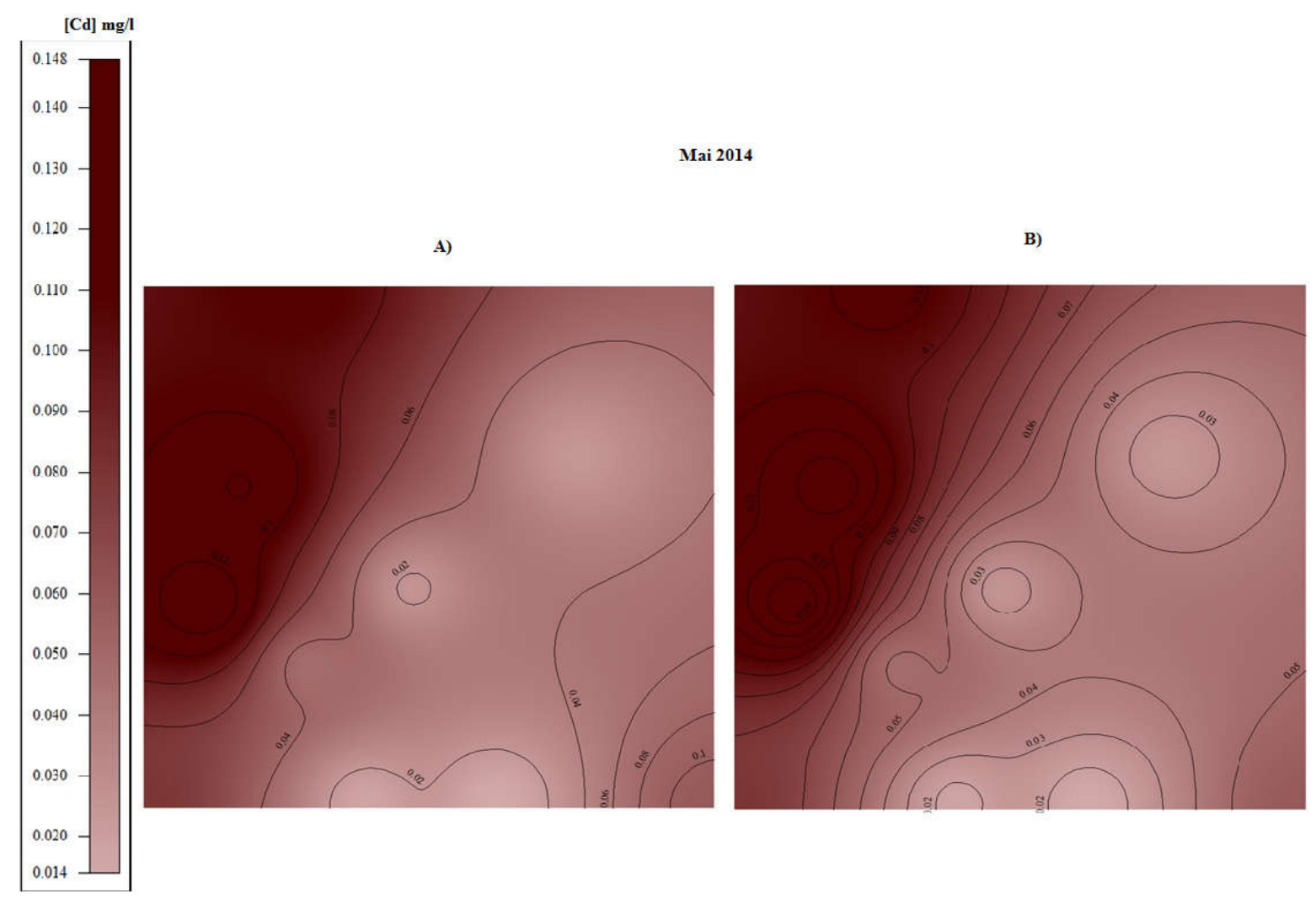

Fig. 10. Distribution spatiale de la concentration du cadmium dans le port de Ténès, Mai 2014 :

A) Surface libre ; B) A $7 \mathrm{~m}$ de profondeur 


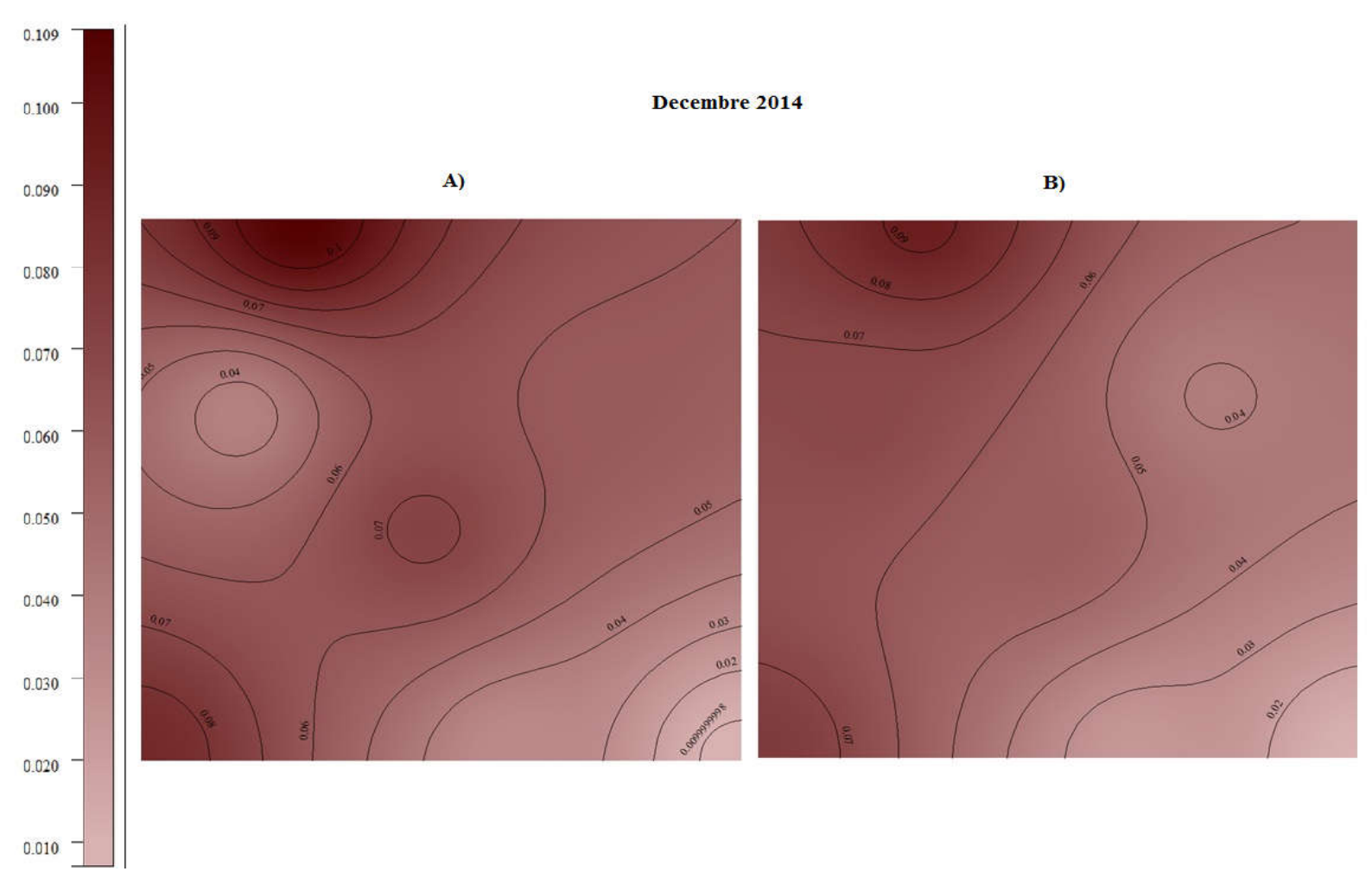

Fig.11. Distribution spatiale de la concentration du cadmium dans le port de Ténès, Decembre 2014 :

A) Surface libre ; B) A $7 \mathrm{~m}$ de profondeur.

Les résultats des analyses sont regroupés dans les figures 10 et 11 où il se dégage que la concentration en $\mathrm{Cd}$ sur la côte est relativement très importante et ce indépendamment de la date du prélèvement. Toutefois, les concentrations moyennes en $\mathrm{Cd}$ pour le mois décembre demeurent plus élevées que celles du mois de mai (4\% de plus). En outre, les concentrations en surface sont plus importantes à travers toute section longitudinale des prélèvements. Ce constat montre qu'il y a une forte dépendance de la concentration en Cd avec :

- En hiver, les particules polluantes sont véhiculées par les cours d'eau provenant des unités industrielles avoisinantes (CERAMIT en particulier).

- Un équilibre d'adsorption-désorption des particules cadmium forme des chlorocomplexes $\left(\mathrm{CdCl}^{+}, \mathrm{CdCl}_{2}\right)$ stables qui passent à l'état de suspension [24].

On note que le cadmium est éliminé au bout d'un temps de résidence de l'ordre de $1.75 \times 10^{5}$ ans [25].

Par biosorption, une partie non négligeable du cadmium se trouve consommée par des 
organismes marins [26]. Pour une analyse récapitulative du comportementdu cadmium, la répartition des différentes espèces chimiques solubles, issues de la dissociation du cadmium, a été établie en fonction de la salinité dans les travaux de Suedel et al 1997 [23].

\section{CONCLUSION}

L'évaluation du degré de la pollution dans le port de Ténès à l'aide des prélèvements spatio-temporels d'échantillons d'eau de mer a été effectuée en surface et en profondeur durant deux périodes (mai et décembre 2014). La qualité de l'eau de mer au port de Ténès, a été contrôlée par la détermination des paramètres hydrologiques (oxygène dissous, température et salinité), de nutriments (nitrites, nitrates) et de métaux lourds (plomb et cadmium). Les résultats obtenus montrent que la solubilité de l'oxygène dépend aussi bien de la température que de la salinité tout en confirmant la validité de l'équation de Benson et de Krauss. En effet, pour une température de $17^{\circ} \mathrm{C}$ et une salinité de $33 \%$, la valeur de l'O.D relevée est de $6.47 \mathrm{ppm}$ laquelle est sensiblement proche de celle donnée par l'équation de Benson et Krauss (=7,63 ppm). Or, l'accroissement de l'activité biologique, pour des températures élevées, a conduit à une sous-saturation du milieu en oxygène dissous. De tels constats corroborent la valeur de $1.46 \mathrm{ppm}$ à $\mathrm{T}=22^{\circ} \mathrm{C}$, laquelle s'écarte substantiellement de celle prévue par l'équation.

Cependant, indépendamment des périodes de prélèvement, de basses concentrations en composés azotés sont obtenues (nitrites $\left[\mathrm{NO}_{2}{ }^{-}\right]<0,08 \mathrm{mg} / \mathrm{l}$ ) et (nitrates $\left[\mathrm{NO}_{3}{ }^{-}\right]<0,25 \mathrm{mg} / 1$ pour mai ; $\left[\mathrm{NO}_{3}{ }^{-}\right]<0,065 \mathrm{mg} / \mathrm{l}$ pour décembre). Ces faibles teneurs indiquent une assimilation des nitrates et nitrites par le biote aquatique ce qui génère une prolifération d'algues et d'eutrophisation dans l'eau de mer. Pour la pollution liée aux métaux lourds spécialement, la concentration en cadmium est élevée au bord de la mer $(=0,384 \mathrm{mg} / \mathrm{l})$ et ceci indépendamment de la date du prélèvement. Pour le plomb, avec ses diverses formes moléculaires (libre ou associé), sa concentration est assez importante et elle dépasse de 60 fois la valeur critique. Cette concentration élevée au fond de la mer $(=4,549 \mathrm{mg} / \mathrm{l})$ est partiellement corrélée avec sa forte présence dans l'environnement. La contamination de l'écosystème marin du port de Ténès est due à une cadence des rejets industriels et humains 
non contrôlés. De nouvelles dispositions législatives doivent être promulguées alors que des procédés appropriés de traitement sont à mettre en place imminement pour réduire l'impact de cette pollution.

\section{REFERENCES}

[1] Nichane M, Khelil M.A. Changements climatiques et ressources en eau en Algérie Vulnérabilité, impact et stratégie d'adaptation. Larhyss Journal. 2015,21, 15-23.

[2] Laib E, Leghouchi E. $\mathrm{Cd}, \mathrm{Cr}, \mathrm{Cu}, \mathrm{Pb}$, and $\mathrm{Zn}$ concentrations in Ulvalactuca, Codium fragile, Janiarubens, and Dictyotadichotoma from Rabta Bay, Jijel (Algeria). Envir Monito and Assess. 2012,184(3),1711-1718.

[3] Belabed BE, Laffray X, Dhib A, Fertouna-Belakhal M, Turki S, Aleya L. Factors contributing to heavy metal accumulation in sediments and in the intertidal musselperna in the Gulf of Annaba (Algeria). Mar Poll Bull. 2013, 74(1), 477-489.

[4] Tayeb A, Chellali M, Hamou A,Debbah S. Impact of urban and industrial effluents on the coastal marine environment in Oran, Algeria. Mar Poll Bull.2015,98(1-2), 281-288.

[5] Kouidri M, Dali youcef N, Benabdellah I, Ghoubali R, Bernoussi A, Lagha1 A. Enrichment and geoaccumulation of heavy metals and risk assessment of sediments from coast of Ain Temouchent (Algeria). Arab J of Geosciences. 2016,3, 354-363.

[6] Benmecheta A, Belkhir L. Oil Pollution in the Waters of Algeria A. Carpenter and A.G. Kostianoy (eds), Oil Pollution in the Mediterranean Sea: Part II - National Case Studies, HdbEnvChem, Springer International Publishing Switzerland. 2016.

[7] Meilleur MA, Wither AW, Coates S. Dissolved oxygen as a physico-chemical supporting element in the Water Frame work Directive. Mar Poll Bull. 2007, 55 (1-6), 53-64.

[8] Bensoussan N, Romano JC, El-Haikali B, Lafont MG. Importance du choixdes échelles de temps pertinentes pour l'étude du fonctionnement d'écosystèmes marinslittoraux. Rend Geosci Comp.2004, 336 (10), 909-918.

[9] Houma F, Touahria T, Bachari NE,Belkessa R. Intégration des données multisources pour la modélisation de la couleur des eaux marines côtières: application à la surveillance du phytoplancton marin. Rapport Commission internationale Mer Méditerranée. 2010,39. 
[10] Ronnie N, Glud.Oxygen dynamics of marine sediments. Mar Biol Res. 2008, 4, 243-289.

[11] Benson BB, Daniel KJr. The concentration and isotopic fractionation of oxygen dissolved in freshwater and seawater in equilibrium with the atmosphere. Limnol Ocean. 1984, 29 (3), 620-632.

[12] Debelius B, Gomez.-Parra A,Forja JM. Oxygen solubility in evaporated seawater as a function of temperature and salinity.Hydro. 2009,632 (1), 157-165.

[13] Conseil canadien des ministres de l'environnement. 1999. Recommandations canadiennes pour la qualité des eaux : protection de la vie aquatique — oxygène dissous (eau de mer), dans Recommandations canadiennes pour la qualité de l'environnement, 1999, Winnipeg, le Conseil.

[14] Jorgensen SE, Gromiec MJ.Mathematical Submodels in Water Quality Systems.Développements in Environmental Modelling.ELSVIER 14. 1989.

[15] Houma F, Bachari NE, Bachouche S, Kassar A, Belkessa. R. PARALIA : Contribution à l'évaluation de la pollution des eaux marines sur la côte Ouest d'Alger en utilisant l'imagerie satellitale. http://www.paralia.fr .2011, 071, 339-344.

[16] Melinder A, Ignatowicz M. Properties of seawater with ice slurry use in focus. Inter J of Refrig, 2015, 52, 51-58.

[17] Tandia AA, Diop ES, Gaye CB. Pollution par les nitrates des nappes phréatiques sous environnement semi-urbain Assaini non: exemple de la nappe de Yeumbeul, Sénégal. J of Afri Earth Sci.1999,29(4), 809-822.

[18] Robertson GP, Groffman PM. Nitrogen transformations, in E.A Paul (ed). In Soil microbiology, ecology and biochemistry. 4th edn Academic Press, Burlington, Massachusetts, USA, 2015, pp. 421-446.

[19] Soleta JL, Renaultb P, Denysc JC, Teuléc G, Dennemontc RM, Domonteb F, Garnierb C, Auberta L, Filleula L, Polycarped D. Discovery and follow-up of a lead-oisoning outbreak in a shantytown of Le Port, Reunion Island, Rev d'épidé et desanté publi. 2013, 61 (4), 329-337. [20] Briant N, Bancon-Montigny C, Elbaz-Poulichet F, Freydier R, Delpoux S, Cossa D. Trace elements in the sediments of a large Mediterranean marina (Port Camargue, France): Levels and contamination history. Mar Poll Bull. 2013, 73 (1), 78-85. 
[21] Gierloff-Emden HG, Hojerslev NK, Krause G, Peters H, Siedler G, Weichart G, Wille P. $\begin{array}{llll}\text { Chemical composition } & \text { of } & 1986\end{array}$ http://link.springer.com/chapter/10.1007/10201933_107 May 14, 2016. 18h:20.

[22] Nicolas B, Bancon-Montigny C, Elbaz-Poulichet.F, Freydier.R, Delpoux S ,Cossa.D Trace elements in the sediments of a large Mediterranean marina (Port Camargue, France): Levels and contamination history. Mari. Poll. Bull. 2013, 73, (1), 78-85.

[23] Achard-Joris M, Gonzalez P, Marie V, Baudrimont M, Bourdineau JP. Cytochrome c oxydase subunit I gene is up-regulated by cadmium in freshwater and marine bivalves. Biometals. 2006,19(3), 237-244.

[24] Noegrohati S. Sorption-Desorption Characteristics of Heavy Metals And Their Availability From The Sediment of SegaraAnakan Estuary. Indo. J. Chem. 2005, 5 (3), 236 244.

[25] Simpson WR.A critical eview of cadmium in the marine environment. Progresin Ocea. $198110(1), 1-70$.

[26] Jakimska A, Konieczka1 P, Skóra K, Namieśnik J. Bioaccumulation of Metals in Tissues of Marine Animals, Part I: the Role and Impact of Heavy Metals on Organisms, Pol J Environ Stud. 2011, 20, (5), 1117-1125

\section{How to cite this article:}

Guetarni F, Douani M, Kouadri Moustefai S.Contribution of anthropic activities to the seawater pollution at the port of Ténès. J. Fundam. Appl. Sci., 2017, 9(3), 1413-1434. 